\title{
The Chronotype of Elite Athletes
}

\author{
by \\ Michele Lastella ${ }^{1}$, Gregory D. Roach ${ }^{1}$, Shona L. Halson ${ }^{2}$, Charli Sargent ${ }^{1}$
}

The aims of this study were (i) to compare the chronotype distribution of elite athletes to a young adult population and (ii) to determine if there was a tendency for athletes to select and/or participate in sports which suited their chronotype. A total of 114 elite athletes from five sports (cricket, cycling, hockey, soccer and triathlon) participated in this study. The participants' chronotype, sleepiness, sleep satisfaction and sleep quality were determined using the Horne and Östberg Morningness and Eveningness questionnaire, the Epworth Sleepiness Scale and questions concerning their sleep satisfaction and quality. All questionnaires were administered during a typical training phase that was not in the lead up to competition and/or post competition. No differences between chronotype group for sleepiness, sleep satisfaction or sleep quality were found. There was a significantly higher proportion of triathletes that were morning and intermediate types compared to the control group $\chi 2(2)=7.5, p=0.02$. A significant relationship between sport and chronotype group $(\chi 2(4)=15.9, p=0.04)$ was observed, with a higher frequency of morning types involved in sports that required morning training. There was a clear indication that athletes tended to select and pursue sports that suited their chronotype. This was evident by the amount of morning types involved in morning sports. Given that athletes are more likely to pursue and excel in sports which suit their chronotype, it is recommended that coaches consider the athlete's chronotype during selection processes or if possible design and implement changes to training schedules to either suit the athletes' chronotype or the timing of an upcoming competition.

Key words: sleep, circadian preference, sport.

\section{Introduction}

Humans typically show large interindividual differences in the timing of their behaviours (e.g. social outings, diurnal activities and sleep habits) (Roenneberg et al., 2003). This is evident in their preferred timing of sleep and wakefulness. At one end of the scale there are morning types and at the other end there are evening types, while in the middle there are intermediate types (Adan et al., 2012). Morning types are phase advanced demonstrating clear preferences in waking up and performing activities in the early morning and find it difficult to remain awake beyond their usual bedtime (Adan et al., 2012). In contrast, evening types are phase delayed such that they prefer going to bed later and find it difficult to wake up early (Adan et al., 2012).

The natural light/dark cycle and normal schedules tend to suit the lifestyle of morning types, such that individuals with a morning preference maintain regular or less irregular sleep/wake schedules compared to individuals with an evening preference (Roepke and Duffy, 2010). Data exploring the chronotype distribution among the general adult population $(19-31$ years) indicate that most individuals are intermediate types $(70 \%)$ with small distributions among morning types (14\%) and evening types (16\%) (Rosenthal et al., 2001).

Few studies have explored the

1 - Central Queensland University, Appleton Institute for Behavioural Science, Adelaide, AUSTRALIA.

2 - Department of Physiology, Australian Institute of Sport, Belconnen, AUSTRALIA. 
chronotype of elite athletes (Samuels, 2008; Silva et al., 2012). In these studies, the majority of athletes were classified as morning types (51\%) and intermediate types (40\%) with a small percentage classified as evening types (9\%). However, these data were derived from school and Paralympic athletes involved in individual sports (i.e., bobsleigh, skeleton, track and field) and thus, may not be representative of elite athlete populations. Silva et al. (2010) revealed that $83 \%$ of athletes presented with daytime sleepiness, $71 \%$ of which were morning types. This is interesting, as previous studies had indicated that evening types typically reported greater daytime sleepiness than morning types (Clodore et al., 1990; Volk et al., 1994). In elite sport, athletes' sleep/wake behaviours are often dictated by their training schedules (Lastella et al., 2015; Sargent et al., 2014). The combination of chronotype and training schedules must correspond for an athlete to train and perform optimally (Drust et al., 2005).

Preliminary findings examining chronotype and batting performance in professional baseball players have indicated that morning types had a higher batting average than players who were evening types in early games (i.e. before 14:00 h). Likewise, evening types had higher batting averages than morning types in later games (i.e. after 20:00 h) (Winter et al., 2011). These data raise important questions concerning the self-selection of sports among elite athletes. For example, athletes involved in sports that require morning training (golf, shooting, triathlon) are mostly morning types, while athletes practising sports that require training in the afternoon or evening (e.g. water polo, volleyball) tend to be evening types (Lastella et al., 2010; Zani et al., 1984). Although these data represent few sports, it is plausible that athletes tend to select, pursue and excel in sports which suit their chronotype. To be explicit, athletes participating in morning sports will be predominantly morning types and athletes participating in evening sports will be evening types. The aims of this study were to (i) compare the chronotype distribution of elite athletes to adults from the general population and (ii) determine if there was a tendency for athletes to select and/or participate in sports which suited their chronotype.

\section{Material and Methods}

\section{Participants}

A total of 88 elite male athletes (age $21.9 \pm 3.8$ years; body height $180.8 \pm 6.0 \mathrm{~cm}$; body mass 73.5 $\pm 8.4 \mathrm{~kg}$ ) and 26 elite female athletes (age $22.6 \pm 4.1$ years; body height $167.2 \pm 6.5 \mathrm{~cm}$; body mass 61.2 $\pm 6.7 \mathrm{~kg}$ ) from five sports (i.e. cricket, 19 males, cycling, 34 males, hockey, 19 females, soccer, 19 males and triathlon, 7 females and 16 males) volunteered to participate in this study. The typical timing of training was employed to categorise each sport as either a morning sport (i.e. AM; cycling, triathlon) or an evening sport (i.e. PM; cricket, hockey, soccer). Participants competing at a national and/or international level were recruited by the physiology staff at the Australian Institute of Sport. Data were compared between athletes $(n=114)$ and an age matched adult population $(n=82)$ recruited as a control group. Participants from the control group were all male with the mean age of $23.4 \pm 4.2$ years (body height $176.4 \pm 7.3 \mathrm{~cm}$; body mass $69.8 \pm 8.3$ $\mathrm{kg})$. The study was approved by the ethics committees of the University of South Australia and the Australian Institute of Sport. All participants provided written informed consent.

\section{Morningness-Eveningness Questionnaire}

Chronotype was evaluated using the Horne and Östberg (1976) Morningness and Eveningness Questionnaire (MEQ). The MEQ is a self-assessment questionnaire which categorizes individuals based on their preference toward performing particular activities in the morning or evening (e.g. If you had no commitments the next day and were entirely free to plan your own day, what time would you get up?). The questionnaire consists of 19 questions and yields scores ranging from 16 to 96 with lower scores indicating participants' preference toward evening activities and higher scores indicating participants' toward morning activities. Chronotype scores were determined using the Horne and Östberg classification system (16-41 = evening type; $42-58$ = intermediate type; 59-86 = morning type) .

\section{Epworth Sleepiness Scale}

The Epworth Sleepiness Scale (ESS) is a scale used to assess daytime sleepiness (Johns, 1991). The ESS asks the participant to rate his or her probability of falling asleep in eight different situations (e.g. sitting and reading; watching $T V$ ) on a scale ranging from 0 (no chance of dozing) to 3 
(high chance of dozing). The scores for the eight questions are added to obtain a single number. A number in the range $0-9$ is considered to be normal while a number in the range 10-24 indicates excessive sleepiness and medical advice should be recommended.

\section{Subjective Sleep Satisfaction and Sleep Quality}

Participants were asked to rate how satisfied they were with the amount of sleep they normally obtained on a Likert scale from 1 (very dissatisfied) to 10 (very satisfied). In addition, participants were asked to rate their normal quality of sleep on a Likert scale from 1 (very poor) to 6 (excellent).

\section{Procedure}

All questionnaires were administered in periods during which the athletes were involved in a typical training phase. This typical training phase was not a period leading up to, or following competition. Athletes were asked to complete questionnaires with regard to sleep, sleepiness and a chronotype at their convenience to avoid collecting data at physically and emotionally stressful moments after training (Silva et al., 2012).

\section{Data Analysis}

Three separate mixed-effects analyses of variance (ANOVA) were conducted to determine the impact of chronotype on the dependent variables (i.e. daytime sleepiness, subjective sleep satisfaction and subjective sleep quality). Each mixed-ANOVA included 'chronotype' (2 levels; morning type, intermediate type) as a fixed term and 'participant' $(n=93)$ as a random term. Evening types were excluded in the mixed-
ANOVAs as only seven athletes were evening types. The statistical significance of all fixed effects was determined using $\mathrm{F}$ tests. The denominator degrees of freedom for $\mathrm{F}$ statistics were computed using the Satterthwaite approximation method. Bonferroni corrections were made to reduce the chances of obtaining a Type 1 error.

Chi square analyses were conducted to (i) examine the chronotype distribution of each sport compared to the chronotype distribution of a group of young adults and (ii) examine the relationship between sport and chronotype groups (morning type, intermediate type). Evening types were excluded in Chi square analyses as only seven athletes were evening types. Data were analysed using SPSS (v17.0) statistical software.

\section{Results}

The mean chronotype score for all athletes was $55 \pm 8$ (range; 32 to 74 ). Using the classification system of Horne and Östberg (1976), 74 participants were intermediate types, 33 participants were morning types and seven participants were evening types. Mixed-effects analysis of variance revealed no significant differences between the morning types, intermediate types or evening types for subjective daytime sleepiness, sleep satisfaction or sleep quality (Table I).

\begin{tabular}{|c|c|c|c|c|c|}
\hline \multicolumn{6}{|c|}{$\begin{array}{c}\text { Table } \mathbf{1} \\
\text { Comparisons between sleepiness, satisfaction with sleep and sleep quality according to } \\
\text { chronotype. }\end{array}$} \\
\hline & \multicolumn{3}{|c|}{ Chronotype } & \multicolumn{2}{|c|}{ Test statistics } \\
\hline Characteristic & $\begin{array}{l}\text { Morning }(n=33) \\
\quad(\text { mean } \pm s)\end{array}$ & $\begin{array}{l}\text { Intermediate }(n=74) \\
\quad(\text { mean } \pm s)\end{array}$ & $\begin{array}{l}\text { Evening }(n=7) \\
\quad(\text { mean } \pm s)\end{array}$ & $\mathrm{F}$ & $p$ \\
\hline Epworth Sleepiness & $8.5 \pm 4.0$ & $8.2 \pm 3.5$ & $5.4 \pm 3.5$ & 2.6 & 0.1 \\
\hline Sleep satisfaction & $7.3 \pm 1.5$ & $6.8 \pm 1.6$ & $6.0 \pm 1.4$ & 3.1 & 0.08 \\
\hline Sleep quality & $4.3 \pm 0.8$ & $4.1 \pm 0.9$ & $3.3 \pm 0.5$ & 0.7 & 1.00 \\
\hline
\end{tabular}


Table 2

Comparisons between athlete representing different sports disciplines and young adults according to chronotype

\begin{tabular}{|c|c|c|c|c|c|}
\hline \multirow[b]{2}{*}{ Characteristic } & \multicolumn{3}{|c|}{ Chronotype } & \multicolumn{2}{|c|}{$X^{2} d f(2)$} \\
\hline & Morning & Intermediate & Evening & $F$ & $p$ \\
\hline Cricket $(n=19)$ & $11 \%$ & $73 \%$ & $16 \%$ & 1.4 & 0.48 \\
\hline Hockey $(n=19)$ & $26 \%$ & $63 \%$ & $11 \%$ & 0.2 & 0.89 \\
\hline Soccer $(n=19)$ & $11 \%$ & $84 \%$ & $5 \%$ & 1.8 & 0.38 \\
\hline Cycling $(n=34)$ & $38 \%$ & $59 \%$ & $3 \%$ & 4.3 & 0.11 \\
\hline Triathlon $(n=23)$ & $48 \%$ & $52 \%$ & $0 \%$ & 7.5 & $0.02^{*}$ \\
\hline Control $(n=82)$ & $22 \%$ & $68 \%$ & $10 \%$ & - & - \\
\hline
\end{tabular}

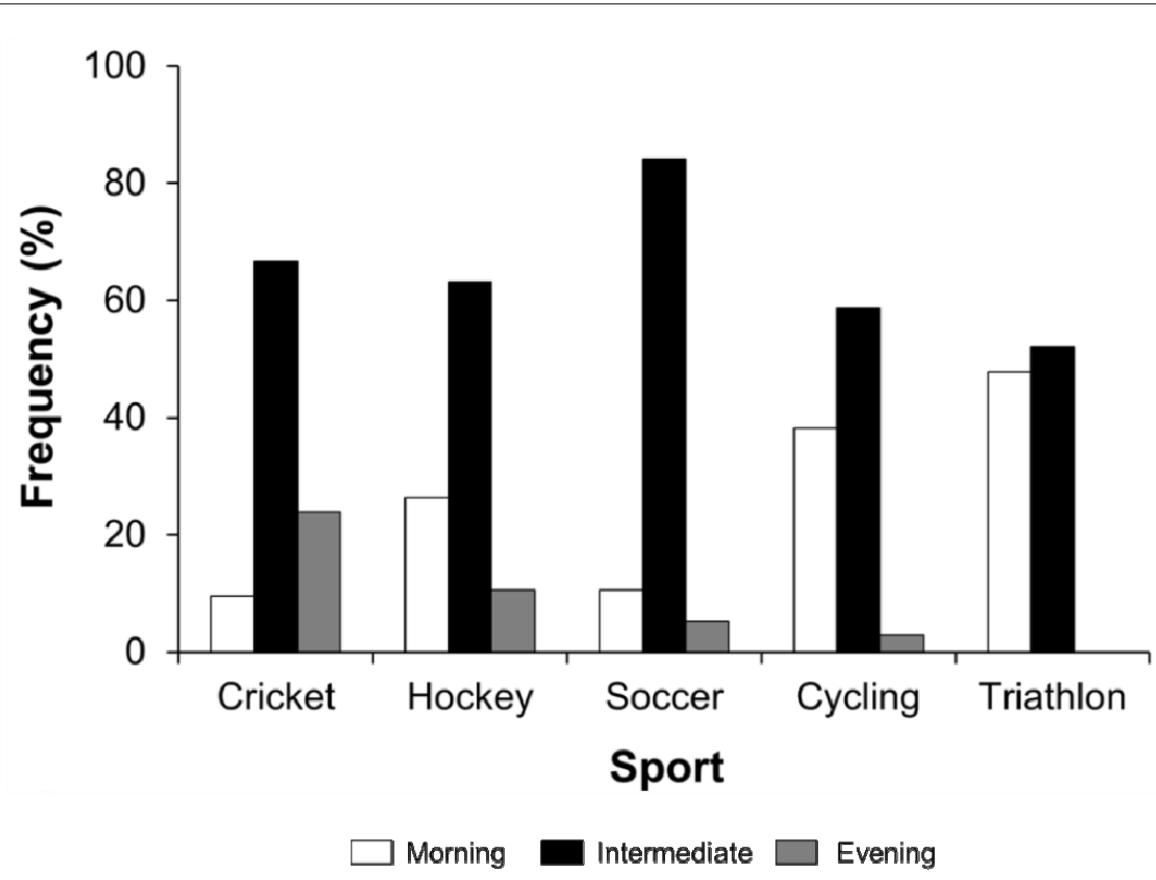

Figure 1

Frequency distribution of athletes' chronotype according to sport 
There were no differences in the chronotype distribution of athletes from cricket, hockey, soccer and cycling compared to the chronotype distribution of the control group (Table II). A significantly higher proportion of triathletes that were morning and intermediate types compared to the control group was found $(\chi 2(2)=7.5, p=0.02)$. There was also a significant relationship between sport and chronotype group $(\chi 2(4)=15.9, \mathrm{p}=0.04)$, with a higher proportion of morning types involved in morning sports. Figure 1 shows the categorisation of chronotype according to sport.

\section{Discussion}

The main findings of this study were that (i) athletes involved in morning sports tended to be either morning or intermediate types and (ii) there were very few athletes classified as evening types with only one evening type athlete involved in a morning sport. This supports the notion that athletes tend to pursue and excel in sports that match their chronotype.

There were no differences in subjective daytime sleepiness, sleep satisfaction or sleep quality between chronotype groups. This finding was contrary to the observations made by Silva et al. (2010), where $71 \%$ of morning types presented with excessive daytime sleepiness. In this sample, only $9 \%$ of morning types reported excessive daytime sleepiness. It is possible that the timing of data collection may have influenced the levels of sleepiness (Silva et al., 2012). For example, Silva et al. (2010) collected data during the preparation for the 2008 Beijing Olympics, whereas the data from the present study was collected during a typical training phase that was not in the lead up to competition and/or post competition. These data indicate athletes are more likely to experience daytime sleepiness during intensive training and competition schedules. This is an important issue as a lack of sleep may have an effect on an athlete's perceived mood and exertion which could impair their motivation, reaction time and performance (Belenky et al., 2003; Blumert et al., 2007; Oliver et al., 2009).

Out of 114 athletes, $28 \%$ were classified as morning types with a high percentage $(72 \%)$ of morning types involved in morning sports. Interestingly, there was only one evening type involved in a morning sport. There are three plausible explanations for this finding. First, and perhaps the most relevant explanation is that elite athletes may pursue sports which suit their chronotype, as individuals typically select activities that match their sleep/wake preferences. For example, morning types are more likely to select and participate in sports that require morning training whereas evening types are more likely to select and participate in sports that require training in the evening (Kunorozva et al., 2012; Rae et al., 2015; Zani et al., 1984). Second, athletes involved in morning sports may have adapted to the early morning training schedules (Rae et al., 2015) and third, athletes involved in morning sports who are evening types may not have progressed from sub-elite or amateur levels as the typical training schedule for morning sports may serve as an additional challenge for evening types attempting to train and perform at a time of day which is opposed to their chronotype.

The idea that individuals differ in the timing of their behaviours (e.g. life habits, diurnal activities, preferred working hours, sleeping habits) is not a recent concept (Horne and Ostberg, 1976; Taillard et al., 2003). For the elite athlete, behaviours such as sleep as well as daytime activities are often dictated by training and competition schedules (Sargent et al., 2014). In circumstances where athletes are required to perform at a different time of day, performance adaptations can occur when training programs are carried out at the time of day at which their upcoming competition will take place (Chtourou et al., 2012; Hill et al., 1989; Souissi et al., 2002). Chtourou et al. (2012) confirmed that exercise training modified the diurnal variations of performance with greater improvement in performance at the time of day at which training was conducted.

The timing of training and competition schedules may affect athletes differently. Athletes participating in a sport which suits their chronotype (e.g. morning type participating in a morning sport) are more likely to train and perform optimally compared to athletes participating in a sport that contradicts their chronotype (e.g. evening type participating in a morning sport). Studies conducted with elite and lower level athletes have demonstrated that the elite athletes' chronotype influences performance, 
whereas chronotype had no influence on performance in lower level athletes (Rossi et al., 1983; Winter et al., 2011). Winter et al. (2011) demonstrated that morning types had higher batting averages than evening types in early games (i.e. before 14:00 h), while evening types had higher batting averages than morning types during evening games (i.e. after 20:00 h). It is reasonable to suggest that athletes involved in sports or performing at a time which suits their chronotype have a better chance of succeeding in that particular sport.

It is acknowledged that there were no performance assessments or analyses conducted on the gender distribution of chronotype within this study. It is important for future investigations to examine the distribution of chronotype between male and female athletes. In addition, further research is required to clarify the influence chronotype has on performance. There is a clear indication that athletes tend to select sports that suit their chronotype, as indicated by the amount of morning types involved in morning sports. Given that athletes are more likely to pursue and excel in sports which suit their chronotype, it is recommended that coaches consider the athlete's chronotype during selection processes or if possible design and implement changes to training schedules to either suit the athlete's chronotype or the timing of an upcoming competition.

\section{Acknowledgements}

This study was financially supported by the Australian Research Council and the Australian Institute of Sport. The authors would like to thank all the players and coaches who took part in the study.

\section{References}

Adan A, Archer SN, Hidalgo MP, Di Milia L, Natale V, Randler C. Circadian typology: A comprehensive review. Chronobiol Int, 2012; 29(9): 1153-1175

Belenky G, Wesensten NJ, Thorne DR, Thomas ML, Sing HC, Redmond DP, Russo MB, Balkin TJ. Patterns of performance degradation and restoration during sleep restriction and subsequent recovery: a sleep dose-response study. J Sleep Res, 2003; 12(1): 1-12. doi: 10.1046/j.1365-2869.2003.00337.x

Blumert P, Crum AJ, Ernstrung M, Volek JS, Hollander DB, Haff EE, Haff GG. The acute effects of twentyfour hours of sleep loss on the performance of national-caliber male collegiate weightlifters. J Strength Cond, 2007; 21(4): 1146-1154

Chtourou H, Chaouachi A, Driss T, Dogui M, Behm DG, Chamari K, Souissi N. The effect of training at the same time of day and tapering period on the diurnal variation of short exercise performances. $J$ Strength Cond, 2012; 26(3): 697-708

Clodore M, Benoit O, Foret J, Bouard G. The Multiple Sleep Latency Test: Individual variability and time of day effect in normal young adults. Sleep, 1990; 13(5): 385-394

Drust B, Waterhouse J, Atkinson G, Edwards B, Reilly T. Circadian Rhythms in Sports Performance-an Update. Chronobiol Int, 2005; 22(1): 21-44

Hill D, Cureton K, Collins M. Circadian specificity in exercise training. Ergonomics, 1989; 32(1): 79-92

Horne JA, Ostberg O. A self-assessment questionnaire to determine morningness-eveningness in human circadian rhythms. Int J Chronobiol, 1976; 4(2): 97-110

Johns MW. A new method for measuring daytime sleepiness: the Epworth sleepiness scale. Sleep, 1991; 14(6): 540-545

Kunorozva L, Stephenson KJ, Rae DE, Roden LC. Chronotype and PERIOD3 Variable Number Tandem Repeat Polymorphism in Individual Sports Athletes. Chronobiol Int, 2012; 29(8): 1004-1010

Lastella M, Roach GD, Hurem D, Sargent C. Does chronotype affect elite athletes' capacity to cope with the 
training demands of elite triathlon. Living in a 24/7 world: The impact of circadian disruption on sleep, work and health Australasian Chronobiology Society, Adelaide, Australia, 2010; 24(7): 25-28

Lastella M, Roach GD, Halson SL, Martin DT, West NP, Sargent C. Sleep/wake behaviour of endurance cyclists before and during competition. J Sports Sci, 2015; 33(3): 293-299

Oliver SJ, Costa RJS, Laing SJ, Bilzon JLJ, Walsh NP. One night of sleep deprivation decreases treadmill endurance performance. Eur J Appl Physiol, 2009; 107(2): 155-161

Rae DE, Stephenson KJ, Roden LC. Factors to consider when assessing diurnal variation in sports performance: the influence of chronotype and habitual training time-of-day. Eur J Appl Physiol, 2015; 115(6): 1339-1349

Roenneberg T, Wirz-Justice A, Merrow M. Life between Clocks: Daily Temporal Patterns of Human Chronotypes. J Biol Rhythms, 2003; 18(1): 80-90

Roepke SE, Duffy JF. Differential impact of chronotype on weekday and weekend sleep timing and duration. Nat Sci Sleep, 2010; 2: 213-220

Rosenthal L, Day R, Gerhardstein R, Meixner R, Roth T, Guido P, Fortier J. Sleepiness/alertness among healthy evening and morning type individuals. Sleep Med, 2001; 2(3): 243-248

Rossi B, Zani A, Mecacci L. Diurnal individual differences and performance levels in some sports activities. Percep Mot Skills, 1983; 57(1): 27-30

Samuels C. Sleep, recovery, and performance: The new frontier in high-performance athletics. Neurol Clin, 2008; 26(1): 169-180

Sargent C, Halson S, Roach GD. Sleep or swim? Early-morning training severely restricts the amount of sleep obtained by elite swimmers. EurJ Sport Sci, 2014; 14 Suppl 1(S1): S310-315. doi: 10.1080/17461391.2012.696711

Silva A, Queiroz SS, Winckler C, Vital R, Sousa RA, Fagundes V, Tufik S, de Mello MT. Sleep quality evaluation, chronotype, sleepiness and anxiety of Paralympic Brazilian athletes: Beijing 2008 Paralympic Games. Br J Sports Med, 2012; 46: 150-154

Souissi N, Gauthier A, Sesboüé B, Larue J, Davenne D. Effects of regular training at the same time of day on diurnal fluctuations in muscular performance. J Sports Sci, 2002; 20(11): 929-937

Taillard J, Philip P, Coste O, Sagaspe P, Bioulac B. The circadian and homeostatic modulation of sleep pressure during wakefulness differs between morning and evening chronotypes. J Sleep Res, 2003; 12(4): 275-282

Volk S, Dyroff J, Georgi K, Pflug B. Subjective sleepiness and physiological sleep tendency in healthy young morning and evening subjects. J Sleep Res, 1994; 3(3): 138-143

Winter W, Potenziano B, Zhang Z, Hammond W. Chronotype as a predictor of performance in major league baseball batters. Sleep, 2011; 34(Suppl)

Zani A, Rossi B, Borriello A. Diurnal interindividual differences in the habitual activity pattern of top level athletes. J Sports Med Phys Fitness,1984; 24(4): 307-310

\section{Corresponding author:}

\section{Michele Lastella}

Central Queensland University, Appleton Institute for Behavioural Science, PO Box 42, Goodwood 5034;

Phone: (0061) 13407956;

E-mail: m.lastella@cqu.edu.au 